\title{
Amizade e Bem-Estar Subjetivo ${ }^{1}$
}

\author{
Luciana Karine de Souza ${ }^{2}$ \\ Mônica Grace Duarte \\ Universidade Federal de Minas Gerais
}

\begin{abstract}
RESUMO - A amizade contribui para o bem-estar subjetivo (BES), e este estudo buscou analisar a relação entre essas variáveis. Participaram 116 universitários de Belo Horizonte e 116 de Porto Alegre, que responderam aos Questionários McGill de Amizade, escalas PANAS e Escala de Satisfação de Vida. As mulheres apontaram mais satisfação e mais sentimentos positivos com a melhor amizade; a amostra mineira indicou mais sentimentos negativos; a satisfação com a amizade correlacionou positivamente com satisfação de vida e afetos positivos, mas não predisse satisfação de vida. Há uma relação próxima, mas não causal, entre BES e amizade. Uma satisfação conjunta com amizades, família e romance é sugerida pela literatura. A pesquisa no Brasil pode considerar o estudo conjunto desses relacionamentos.
\end{abstract}

Palavras-chave: amizade, bem-estar, adultos

\section{Friendship and Subjective Well-Being}

\begin{abstract}
Friendship contributes to subjective well-being, and this study analyzed the relationship between these variables. 116 university students from Belo Horizonte and 116 from Porto Alegre, both cities in Brazil, responded to the McGill Friendship Questionnaires, the PANAS scales and the Life Satisfaction Scale. Women showed more satisfaction and positive feelings towards their best friend; students from Belo Horizonte reported more negative feelings; friendship satisfaction correlated positively with life satisfaction and positive affect, but did not predict life satisfaction. There was a close, yet not causal, relation between subjective well-being and friendship. Joint satisfaction with friends, family, and romance is necessary for subjective well-being, as suggested by scientific literature. Research in Brazil could direct efforts to joint analyzes of these relationships.
\end{abstract}

Keywords: friendship, well-being, adults

No Relatório de Desenvolvimento Humano Global de 2011, o Brasil consta em 84 lugar no Índice de Desenvolvimento Humano e no quesito satisfação geral com a vida, dentre 187 países (Programa das Nações Unidas para o Desenvolvimento - PNUD, 2013). Essa posição evidencia que o país precisa atentar para questões importantes à felicidade, como o respeito a todos, a validação de direitos, a ampliação de oportunidades. Para além de indicadores objetivos, esses relatórios mostram a preocupação com o aspecto subjetivo da felicidade, por isso é relevante investir na pesquisa psicológica desse campo.

Na literatura psicológica a felicidade vem sendo referida como bem-estar subjetivo (BES), satisfação, estado de espírito, afeto positivo ou avaliação subjetiva da qualidade de vida. Para Myers (2000), felicidade é algo mais profundo e duradouro do que um bom humor momentâneo. Segundo esse autor, pessoas felizes são menos autocentradas, menos hostis e abusivas, menos vulneráveis a doenças, mais amáveis, tolerantes, confiáveis, criativas, decididas e dispostas a ajudar.

1 Apoio: CNPq; FAPEMIG; PRPq-UFMG; FUNDEP/SANTANDER. Agradecimentos a C. S. Hutz, M. Mendelson; aos participantes e docentes que possibilitaram a coleta de dados.

2 Endereço para correspondência: Universidade Federal de Minas Gerais, Faculdade de Filosofia e Ciências Humanas, Departamento de Psicologia, Av. Antônio Carlos, 6627, sala F-4050, Campus Pampulha, Belo Horizonte, Minas Gerais, CEP 31.270-901. E-mail: lukarides@gmail.com
Diener (1984) identifica três tratamentos ao BES na literatura: 1) é concebido por critérios externos, como uma virtude, e a felicidade seria a posse de qualidades desejáveis; 2) abrange o que leva as pessoas a avaliarem positivamente suas vidas; e 3) considera o BES como o estado de preponderância de afetos positivos sobre os negativos. Diener (1984) destaca, ainda, os seguintes aspectos: a subjetividade, uma vez que o bem-estar é parte da experiência individual; o entendimento de que ele é composto por fatores positivos, e não só pela ausência de fatores negativos; e o fato de o bem-estar incluir uma medida global, no lugar de somente uma medida limitada de um único aspecto da vida.

Duas tradições filosóficas fundamentam as diferentes concepções da literatura, como relatam Woyciekoski, Stenert e Hutz (2012). A Tradição Hedônica considera as emoções prazerosas, satisfação de vida e relativa ausência de estados desprazerosos; na Tradição Eudaimonica o bem-estar é visto de modo integral, daí o conceito de bem-estar psicológico presente em muitos estudos (Moreira \& Sarriera, 2006). Raramente são investigados o BES e o bem-estar psicológico juntos (e.g. Freire \& Tavares, 2011).

Uma das formas de tratamento científico mais utilizadas tem sido a proposta de Diener, Lucas, Oishi e Suh (2002). Para eles, BES consiste de três componentes distintos, porém correlacionados: satisfação de vida (avaliação cognitiva global da vida da pessoa), afeto positivo e ausência de afeto negativo. Portanto, BES envolve o que as pessoas pensam e sentem a respeito de suas condições de vida, com critérios de 
avaliação do próprio indivíduo, e não externos. Trata-se de uma grande categoria de fenômenos incluindo as respostas emocionais das pessoas (afeto positivo e negativo), domínios de satisfação e os julgamentos globais de satisfação de vida (Giacomoni, Hutz, \& Reppold, 2007).

Muitos estudos mostram que pessoas felizes (ou seja, felicidade enquanto BES) são bem sucedidas em vários domínios de suas vidas. Woyciekoski et al. (2012) localizaram, na literatura publicada entre 1980 e 2011, que os fatores intrínsecos associados ao BES são personalidade, lócus de controle, confiança, defesas repressivas, otimismo, religiosidade, virtudes e a forma como a pessoa pensa/percebe o mundo. Nível socioeconômico, cultura (individualista ou coletivista), suporte social, relacionamentos (família, cônjuge e amigos) e eventos de vida foram identificados como fatores extrínsecos.

O trabalho de Argyle (2001) em psicologia da felicidade reuniu dados de diversos países sobre aspectos importantes à vida de seus habitantes. Três dimensões da vida trazem mais felicidade às pessoas: trabalho, lazer e relacionamentos.

O papel dos relacionamentos no BES é um interesse recorrente em pesquisa. Ao final da década de 1980, Pavot, Diener e Fugita (1990) concluíram que as pessoas relatam sentimentos de maior felicidade diante de apoio social. Na década seguinte, Myers (2000) também salienta o aspecto interpessoal da felicidade, apontando que pessoas casadas relatam maior felicidade e satisfação de vida do que pessoas que nunca casaram ou que se separaram/divorciaram. Lyubomirsky, King e Diener (2005) sugerem que pessoas felizes, isto é, aquelas que experimentam mais afetos positivos do que negativos, tendem a ter sucesso e mais satisfação com relacionamentos. Woyciekoski et al. (2012) também denotaram essa relação entre BES, apoio social e relacionamentos.

Segundo Argyle (2001), são três os tipos de relacionamentos que mais explicam a felicidade: família, romance e amizade. O foco do presente trabalho está na relação entre amizade e BES. Assim, cabe apontar os estudos que se dedicaram especificamente a estudar o papel da amizade para a felicidade e bem-estar das pessoas. Contudo, previamente é apresentado um breve panorama sobre as relações de amizade.

A amizade é um relacionamento percebido como significativo e que envolve aspectos como companheirismo, ajuda (instrumental, emocional e social), confiança, autorrevelação, proximidade relacional, autovalidação, respeito, lealdade e disponibilidade. Do amigo se espera e a ele se oferece perdão, compaixão, paciência, empatia, altruísmo, gratidão e honestidade (Souza \& Hutz, 2007a).

A amizade difere conforme a etapa de vida. Enquanto na infância ela se vê pautada por brincadeiras, companheirismo, diversão e afeto, na adolescência ela se acresce de lealdade, confiança, proximidade e autorrevelação (Bukowski, Newcomb, \& Hartup, 1996; Peron, Guimarães \& Souza, 2010). É na saída da adolescência e entrada na adultez jovem que as amizades atingirão um ápice funcional desenvolvimental, ajudando o indivíduo em sua adaptação a novas tarefas de vida e desafios em termos de relações interpessoais e de carreira profissional (Rawlins, 1992). No entanto, a quantidade de amigos diminui com a entrada na adultez e o envolvimento em romance estável, casamento, surgimento de filhos e dedicação ao trabalho (Carbery \& Buhrmester, 1998).
Ainda assim, a amizade é um relacionamento importante para a vida adulta, por vezes como complemento à ausência de laços familiares fortes. Dessa forma, cabe investiga-lo na relação com o BES em adultos.

Nas diferenças de gênero na percepção da qualidade da amizade, as mulheres experimentam mais satisfação e sentimentos positivos com relação à melhor amizade do que os homens (Mendelson \& Aboud, 2003; Souza \& Hutz, 2007b). Um estudo com adultos-jovens gaúchos (média de 23 anos) não encontrou diferenças de gênero para sentimentos negativos (Souza \& Hutz, 2007b). Para as funções da amizade, entendidas por Mendelson e Aboud (1999) como ajuda, autovalidação, aliança confiável, companheirismo estimulante, intimidade e segurança emocional, encontrou-se diferença apenas para homens que indicaram uma melhor amiga: para eles, essa amiga é capaz de melhor prover segurança emocional e autovalidação (Souza \& Hutz, 2007b). Ainda assim, prevalecem amizades de mesmo sexo, tanto em estudos brasileiros (DeSousa \& Cerqueira-Santos, 2012; Souza \& Hutz, 2007b) como de outros países (Mendelson \& Aboud, 1999). Estudos complementares com adultos brasileiros mostraram também que os homens não costumam dizer ao melhor amigo que ele tem esse status, bem como, sem distinção de gênero a origem da melhor amizade é a escola e o melhor amigo mora na mesma cidade do participante (Souza \& Hutz, 2008a).

O presente trabalho segue o modelo teórico de Mendelson e Aboud $(1999,2003)$ para a qualidade da amizade (seis funções, sentimentos positivos e negativos e satisfação com a amizade). Esses construtos são avaliados pelos Questionários McGill de Amizade, validados para uso no Brasil por Souza e Hutz (2007a) (mais detalhes na seção de procedimentos metodológicos). Ao mesmo tempo em que a presente pesquisa foi conduzida, DeSousa e Cerqueira-Santos (2012) realizaram um estudo com as escalas validadas por Souza e Hutz (2007a) com 124 adultos-jovens do Sergipe (idade média de 23 anos). Confirmaram-se os achados anteriores quanto a um maior número de amizades de mesmo sexo, residentes na mesma cidade, com origem na escola e melhor amizade que conhece esse status. No entanto, os autores não realizaram comparações de sexo para as funções, sentimentos e satisfação com o relacionamento. Portanto, novos estudos com tais análises podem colaborar com o corpo de trabalhos desse campo, inclusive com a introdução da variável BES. A seguir é revisada a literatura com os estudos disponíveis que evidenciam a associação entre amizade e BES.

McDonough e Munz (1994) encontraram, em amostra com 198 adultos, evidência de que elevados níveis de bem-estar se associa a níveis mais profundos de participação nos relacionamentos de amizade em termos de comunicação e consideração, ao passo que baixos níveis de bem-estar se associaram mais à função de companheirismo nas amizades estudadas. Ademais, bem-estar psicológico e físico foram mais altos em pessoas com relacionamentos próximos com família e amigos, o que condiz com a literatura sobre felicidade e relacionamentos.

Rodriguez, Mira, Myers, Morris e Cardoza (2003) realizaram um estudo com 338 adultos-jovens (idade média de 22 anos) de etnia latina residentes nos Estados Unidos com o propósito de avaliar a relação entre apoio percebido de 
amigos e familiares e o bem-estar na redução do estresse. Ainda que ambos os apoios da família e dos amigos contribuíram para sentimentos de bem-estar, o dos amigos foi visto como mais relevante para o bem-estar positivo e redução do estresse. Esse estudo é interessante na medida em que utiliza uma amostra cuja bagagem cultural é composta tanto pela cultura norte-americana como pela latino-americana, e a cultura é um dos determinantes do BES, como indicado por Woyciekoski et al. (2012). Além disso, tratou-se de uma amostra com adultos-jovens, o que indica que a importância da amizade prossegue na entrada para a vida adulta, como apontado também nos estudos empíricos sobre amizade nessa faixa etária (Carbery \& Buhrmester, 1998; Rawlins, 2002).

Demir, Özdemir e Weitekamp (2007) investigaram as relações entre a melhor amizade e amizades próximas e o BES em 280 adultos-jovens (média de 23 anos). Enquanto para mensurar BES os autores utilizaram o modelo de Diener et al. (2002), para avaliar a qualidade da amizade e suas funções foram utilizadas a versão reduzida das escalas de Furman e Buhrmester (1985), abordando companheirismo, intimidade, aliança confiável, afeto e conflito (confiabilidade das escalas entre 0,84 e 0,90 ). As análises mostraram que as características da amizade estudadas para a melhor amizade do participante se associaram positiva e significativamente com o BES (correlações entre 0,25 e 0,70), com exceção de conflitos. Uma análise de regressão foi conduzida, evidenciando que a qualidade da melhor amizade foi capaz de significativamente prever felicidade (BES), e que o companheirismo foi a característica mais responsável por esse resultado. Interessantemente, McDonough e Munz (1994) já haviam mostrado uma relação relevante entre companheirismo e bem-estar.

Ao final do trabalho, todavia, Demir et al. (2007) fazem a ressalva de que as escalas usadas para avaliar a qualidade da amizade no estudo contribuíram para o resultado de que a melhor amizade respondeu por $8 \%$ da variância relativa ao $\mathrm{BES}$, e que outras medidas da qualidade da amizade podem trazer índices melhores. Foi o que mostrou a pesquisa de Demir e Weitekamp (2007), na qual foram utilizadas a escala das funções da amizade de Mendelson e Aboud (1999) e as três escalas de Diener et al. (2002).

Demir e Weitekamp (2007), em um estudo sobre amizade e personalidade como preditores da felicidade (300 mulheres e 123 homens norte-americanos, média de idade de 22,5 anos), encontraram que tanto as funções da amizade como o nível de conflito na amizade (escala criada pelos autores) responderam por $15 \%$ da variância envolvida no resultado de que funções e conflitos na amizade predizem felicidade. Ademais, verificaram que as funções de companheirismo e de autovalidação foram fortes preditores da felicidade, quando foram controladas as variáveis gênero e personalidade. Um estudo com amostra brasileira somaria achados à literatura no que diz respeito à relação amizade e felicidade, com relação aos itens não avaliados, como satisfação com a amizade e sentimentos positivos e negativos com relação ao melhor amigo.

Gilman et al. (2008) analisaram a qualidade de vida em 1338 adolescentes (média de 15 anos) de nações individualistas (Irlanda e Estados Unidos) e coletivistas (China e Coreia do Sul). Americanos e Irlandeses relataram maior satisfação com amigos que os demais adolescentes, e os chineses maiores taxas de satisfação com a família. Interessantemente, Lansford, Antonucci, Akiyama e Takahashi (2005) compararam relacionamentos sociais e bem-estar com adolescentes e adultos dos Estados Unidos e do Japão (faixa etária de 13 a 93 anos) - uma nação individualista e uma coletivista, conforme o estudo de Gilman et al. (2008). Estar casado e/ou ter um melhor amigo associou-se positivamente com bem-estar, ao passo que relacionamento com pais ou com filhos não apresentou associação significativa nos dois países investigados.

Park e Huebner (2005) encontraram correlação positiva entre satisfação global de vida e satisfação com os amigos em adolescentes (média de 15 anos) coreanos e estado-unidenses. Esse resultado também se aproxima ao do encontrado por Gilman et al. (2008) com culturas semelhantes. Importa lembrar que a cultura é listada como um dos fatores determinantes do BES (Woyciekowski et al., 2012).

Embora comumente estudada na comparação entre nações, caberia investigar também possíveis diferenças quanto ao BES e à amizade em grupos culturais de um mesmo país, por exemplo, representados por estados localizados em regiões distintas. Comparações entre estados brasileiros com respeito ao BES poderia trazer novos insights na questão de como pessoas de diferentes regiões do país percebem a qualidade do relacionamento de amizade.

São raros os estudos brasileiros sobre amizade em adultos, e apenas um adotou uma abordagem predominantemente quantitativa com grande amostra na avaliação da qualidade da amizade com instrumentos que detalham funções da amizade, satisfação global com o relacionamento, e sentimentos positivos e negativos direcionados ao amigo (Souza \& Hutz, 2007b). O estudo de DeSousa e Cerqueira-Santos (2012) não verificou diferenças de sexo. Ademais, o único estudo brasileiro localizado sobre amizade e BES foi o de Kipper (2003) com 60 participantes adultos divididos entre aqueles com amizades no trabalho e aqueles que possuem apenas colegas que não são amigos. Para estes últimos, os dados do BES apontaram índices significativamente maiores de afetos negativos e menores de afetos positivos, na comparação com os que possuem colegas que também são amigos. Portanto, seria uma contribuição à literatura científica nacional um estudo que investigue BES e amizade, com amostra maior e sem o entrelaçamento de dois tipos distintos de relacionamentos (de colegas de trabalho e de amizade).

Em suma, a literatura oferece dados mostrando que: são escassos os estudos brasileiros sobre amizade em adultos; há medidas objetivas e consistentes para a avaliação da qualidade da amizade, no caso, considerando afetos positivos e negativos, satisfação global com o relacionamento e funções principais da amizade (Mendelson \& Aboud, 1999, 2003); a amizade é percebida de modo diferente dentre distintos grupos culturais; a investigação de outras variáveis importantes para as relações de amizade são relevantes, como no caso, indicado na literatura, do estado civil; as relações de amizade são importantes para a felicidade das pessoas, no caso, para o BES, com evidências de distintos países; não há estudos nacionais que abordem BES e amizade utilizando ambos os modelos de Mendelson e Aboud (1999, 2003) e de Diener et al. (2002). Como já abordado em estudo brasileiro anterior, 
são exemplos de outras variáveis que poderiam agregar novos achados à pesquisa os seguintes fatores: origem da amizade, duração, relacionamento concomitante, amizades extras, reciprocidade, e características importantes à amizade indicadas espontaneamente pelos participantes. Além disso, o gênero mostra vivências diferenciadas para as amizades e, portanto, deve fazer parte das análises.

O presente trabalho vem procurar responder a mais de uma lacuna na literatura, como argumentado. O objetivo principal da pesquisa foi investigar a relação entre a melhor amizade e BES em adultos-jovens de duas regiões geográficas brasileiras, utilizando-se os modelos de Diener (1984) e de Mendelson e Aboud (1999, 2003). Como objetivos secundários, buscou-se: verificar diferenças de gênero e de estado civil, bem como descrever e comparar características da amizade entre gaúchos e mineiros em termos de origem da melhor amizade, duração, relacionamento concomitante, amizades extras, percepção da reciprocidade no relacionamento, e características da amizade indicadas espontaneamente pelos participantes.

\section{Método}

\section{Participantes}

O estudo contou com 116 participantes de Minas Gerais (MG) (média de 20,2 anos) e 116 do Rio Grande do Sul (RS) (26 anos), de ambos os sexos e níveis socioeconômicos distintos. A amostragem foi por conveniência e com a colaboração de professores da Universidade Federal de Minas Gerais e da Universidade Federal do Rio Grande do Sul.

\section{Instrumentos e Procedimentos}

Os participantes preencheram, coletivamente e durante um período de aula, o Termo de Consentimento Livre e Esclarecido e os instrumentos da pesquisa. O preenchimento levou, em média, 30 minutos. Os instrumentos utilizados foram os Questionários McGill de Amizade (Mendelson \& Aboud, 1999, 2003), validados para uso no Brasil por Souza e Hutz (2007a, 2007b), e as Escalas PANAS (Watson, Clark, \& Tellegen, 1988) e a Escala de Satisfação de Vida (ESV) (Diener, Emmons, Larsen, \& Griffin, 1985), validadas por Giacomoni e Hutz (1997).

A ESV é formada por cinco afirmações relativas à satisfação com a própria vida (exemplo: "Minhas condições de vida são excelentes"); para cada uma, o participante deve marcar como se sente diante de sete pontos em uma escala Likert (entre $1=$ discordo plenamente e $7=$ concordo plenamente). A consistência interna foi de 0,87 (Giacomoni \& Hutz, 1997). As Escalas PANAS oferecem uma lista de 40 adjetivos que representam o estado de humor a que se referem, organizados alfabeticamente, sendo 20 positivos (como em "entusiasmado") e 20 negativos (por exemplo, "nervoso"). A pessoa deve redigir à esquerda de cada adjetivo o número que melhor representa "até que ponto você tem se sentido desta forma ultimamente" (entre $1=$ nem um pouco e $5=$ extremamente). A consistência interna foi de 0,88 e 0,86 , respectivamente, para afetos positivos e negativos (Giacomoni \& Hutz, 1997).

São três os Questionários McGill de Amizade. O Questionário das Funções da Amizade (QFA) avalia seis funções da amizade: ajuda, aliança confiável, autovalidação, companheirismo, intimidade e segurança emocional, representadas por cinco sentenças (total de 30 itens). Para cada uma, o participante deve indicar como se sente em relação ao amigo: 1 = nunca, $2=$ raramente, $3=$ de vez em quando, $4=$ muito frequentemente e $5=$ sempre. A Escala de Satisfação com a Amizade (ESA) avalia o nível de satisfação com o relacionamento com o amigo em sete sentenças; já a Escala de Sentimentos Positivos com relação ao Amigo (ESPA) utiliza seis. São respondidas em conjunto com as opções 1 = discordo muito, 2 = discordo em parte, 3 (sem rótulo), $4=$ concordo em parte, e $5=$ concordo muito. A Escala de Sentimentos Negativos com relação ao Amigo (ESNA) possui 18 sentenças, com as opções de $1=$ nunca, $2=$ raramente, $3=$ de vez em quando, $4=$ frequentemente, e $5=$ muito frequentemente. A consistência interna das escalas que compõem os Questionários McGill variou de 0,73 a 0,89 (Souza \& Hutz, 2007a).

Utilizou-se também um questionário sociodemográfico (sexo, estado civil, naturalidade, cidade de residência, curso universitário) e o Questionário Complementar de Amizade (Souza \& Hutz, 2008a), que trata das questões como origem da melhor amizade, duração, relacionamento concomitante, amizades extras, percepção da reciprocidade no relacionamento e características importantes à amizade (uma questão aberta sobre um aspecto importante da amizade não abordado nos questionários). Para as análises foram conduzidas comparações de médias (testes $t$ ), correlações (Pearson), post hoc de Bonferroni e análise de regressão, mediante o pacote estatístico SPSS.

\section{Resultados}

Serão apresentados primeiramente os dados descritivos sobre as amizades das duas amostras, com base no Questionário Complementar de Amizade. As mulheres mineiras (79\%) indicaram menos amizades de mesmo sexo, na comparação com as gaúchas (84\%). Já o vínculo concomitante com a melhor amizade investigada foi maior com ex-colegas de colégio (32\%) na amostra mineira, enquanto que para a maioria dos gaúchos a melhor amizade não possui outro vínculo concomitante (33\%), ou seja, a melhor amizade não é um atual ou ex-colega de escola, nem de trabalho, nem integrante da família, vizinho ou parceiro romântico - é apenas um(a) amigo(a), sem laços prévios. A média da duração da melhor amizade foi maior para a amostra gaúcha (10 anos) do que para a mineira (aproximadamente 7 anos). Já quanto ao participante saber se a sua melhor amizade tem conhecimento desse status de melhor amigo(a), 64\% dos mineiros indicou sim e $61 \%$ dos gaúchos afirmou que também crêem que sua melhor amizade sabe que está no topo da lista de amigos do respondente.

Para a análise da questão do aspecto da amizade não abordado nos questionários, foi possível analisar um mon- 
Tabela 1. Correlações entre as Dimensões do Bem-Estar Subjetivo e da Amizade

\begin{tabular}{lccc}
\hline Dimensões da Amizade & Satisfação de Vida & Afeto Positivo & Afeto Negativo \\
\hline Satisfação com a Amizade & $0,13^{*}$ & $0,27^{* *}$ & $-0,16^{*}$ \\
Sentimentos Positivos com o Amigo & 0,11 & $0,18^{* *}$ & $-0,1$ \\
Sentimentos Negativos com o & $-0,17^{* *}$ & $-0,03$ & $0,21^{* *}$ \\
Amigo & & -03 & \\
\hline
\end{tabular}

Nota. ${ }^{* *} \mathrm{p}<0,01 ; * \mathrm{p}<0,05$.

tante maior de questionários: 256 questionários coletados em MG e 118 no RS. Desse total $(\mathrm{N}=374), 43, \%$ dos mineiros deixaram uma contribuição nesse sentido, e 38,1\% dos gaúchos colaboraram. Tomando-se as duas amostras em conjunto, a maioria das sugestões foram avaliadas pelos instrumentos. As demais sugestões se assemelham às encontrados por Duarte e Souza (2010) com adultos gaúchos, como afinidades/identificação, irmandade, amizade pela Internet, admiração, respeito, reciprocidade, caráter e virtudes. Os conteúdos novos envolveram o fato de ter familiares ou parceiros românticos como melhor amizade, e a influência bidirecional (positiva ou negativa) de tais relações com a amizade da pessoa.

Na comparação de médias entre homens e mulheres, as mulheres apresentaram significativamente mais satisfação e mais sentimentos positivos direcionados à melhor amizade do que os homens. A média feminina para a satisfação com a amizade foi de 4,79, enquanto a masculina foi de 4,65 ( $t$ $=2,54 ; \mathrm{gl}=230 ; \mathrm{p}=0,012$ ). Nos sentimentos positivos, as mulheres tiveram média de 4,87 , ao passo que os homens tiveram 4,73 $(t=3,56 ; \mathrm{gl}=230 ; \mathrm{p}<0,001)$. A comparação de médias de sentimentos negativos associados ao amigo não encontrou diferença significativa entre mulheres $(1,92)$ e homens $(1,96)$.

Na comparação de médias entre mineiros e gaúchos com respeito à percepção da qualidade da melhor amizade, não foi encontrada diferença estatisticamente significativa para as seis funções da amizade, sentimentos positivos, nem para a satisfação com o relacionamento. Nos sentimentos positivos, a média entre os mineiros foi de 4,81 , enquanto entre os gaúchos foi de 4,84. Na satisfação com a amizade, a amostra de MG obteve média de 4,71, ao passo que a amostra sulista apresentou média de 4,78. A diferença significativa foi nos sentimentos negativos associados ao amigo. Enquanto a amostra mineira indicou média de 2,02, a gaúcha indicou $1,85(t=2,96 ; \mathrm{gl}=230 ; \mathrm{p}=0,003)$.

No BES apenas a dimensão de afeto positivo não apresentou diferença significativa entre sexos. A média nas mulheres foi de 61,8 e nos homens foi de 62,1 . Os homens indicaram mais afeto negativo que as mulheres $(t=-2,27 ; \mathrm{gl}=230$; $\mathrm{p}=0,024)$, com os primeiros indicando média de 47,8 , e as segundas de 44,0. Na satisfação de vida, a diferença foi estatisticamente significativa mas limítrofe $(t=1,93 ; \mathrm{gl}=$ $230 ; \mathrm{p}=0,055)$, com as mulheres tendo média de 24,3 , e os homens de 22,8 .

Nas comparações entre estados e BES, a diferença significativa encontrada foi para afeto positivo $(t=-2,48 ; \mathrm{gl}=$ 229; $\mathrm{p}=0,014)$, com média de 63,9 dos gaúchos, superior aos 59,9 dos mineiros. Para afeto negativo, a média de MG foi de 45,3 , praticamente igual à do RS $(45,2)$. Na satisfação de vida, a média do RS foi 24,3 e a de MG foi de 23,3.
A comparação de BES por estado civil apontou uma diferença estatisticamente significativa $(\mathrm{F}(2,226)=4,24$; $\mathrm{p}=0,015)$ entre participantes casados e outros dois grupos (solteiros e namorando/noivos), segundo o teste post hoc (Bonferroni). A média de afeto positivo foi estatisticamente superior nos casados $(68,5)$ na comparação com solteiros $(60,9)(\mathrm{p}=0,014)$ e com namorando/noivos $(61,4)(\mathrm{p}=$ $0,029)$. Não foram detectadas interações significativas entre as variáveis "estado*sexo*estado civil" para satisfação de vida ou para satisfação com a amizade de acordo com a análise de variância efetuada.

A Tabela 1 apresenta as correlações entre as dimensões do BES e da amizade. Como se pode notar, os sentimentos negativos com relação ao amigo não se relacionam significativamente ao afeto positivo da pessoa, e os sentimentos positivos direcionados à melhor amizade não se correlacionam significativamente com a satisfação de vida nem com o afeto negativo. Todavia estar satisfeito com a vida e sentir-se predominantemente contente está positivamente associado a uma boa satisfação com a melhor amizade, e negativamente associado com afetos negativos. Os sentimentos positivos com o amigo e o afeto positivo estão fortemente relacionados, como seria de se esperar e concordando com as correlações de afeto positivo e satisfação com a amizade.

Como as comparações entre estados geraram diferenças significativas nos afetos positivos do BES e nos sentimentos negativos relacionados ao amigo, decidiu-se realizar análise de regressão separadamente por estado. Também não foram inseridas as funções da amizade dada a diferença de gênero apontada na literatura (Souza \& Hutz, 2007b) e dado que as demais escalas são mais voltadas para avaliações globais relacionadas à amizade (satisfação com o relacionamento e sentimentos positivos e negativos). Após a análise envolvendo BES e as escalas de satisfação com a amizade e de sentimentos com o amigo, foi possível identificar três direcionamentos gerais quanto à felicidade. Para os mineiros, $o$ afeto negativo prediz satisfação de vida $(B=-0,468, t(110)$ $=-5,25, \mathrm{p}<0,01)$. Já quanto à amostra gaúcha, o afeto positivo $(B=0,450, t(111)=5,913, \mathrm{p}<0,01)$ e o afeto negativo $(B=-0,343, t(111)=-4,513, \mathrm{p}<0,01)$ predizem, juntos, a satisfação de vida. Já com respeito à amizade e BES, as análises demonstraram que a satisfação com a amizade não prediz satisfação de vida, tampouco unindo as amostras ou adicionando/retirando variáveis do modelo.

\section{Discussão}

Foram realizadas análises para melhor compreender a relação entre a melhor amizade e BES em adultos-jovens de duas regiões geográficas brasileiras. Em complemento, 
foram investigadas diferenças de gênero e de estado civil, bem como empreendidas comparações de características da amizade entre gaúchos e mineiros.

Nas comparações de gênero na amizade, as mulheres apresentaram maior satisfação com a melhor amizade e mais sentimentos positivos direcionados a ela. Esse resultado é semelhante ao apontado por Mendelson e Aboud (1999, 2003) e pelo estudo de Souza e Hutz (2007b) com adultos gaúchos. Dessa forma, realmente parece haver uma consistência de resultados que confirmam a percepção das mulheres sobre suas melhores amizades como de satisfação mais elevada e de vivência de mais sentimentos positivos do que no caso dos homens e suas melhores amizades. A recomendação que Wright (1988) faz, contudo, é de que muitas vezes os modelos teóricos para amizade utilizam como base a experiência feminina com amizades, adotando, portanto, um viés sexista no estudo desses relacionamentos. Ainda assim, futuros estudos sobre amizade em adultos já podem partir de análises separadamente por gênero, aprofundando outras variáveis e aspectos mais qualitativos da satisfação com a amizade e dos sentimentos positivos.

No entanto, os sentimentos negativos não diferenciaram entre homens e mulheres, da mesma forma que no estudo nacional prévio (Souza \& Hutz, 2007b). De um lado, cabe considerar que, como se trata do segundo estudo brasileiro em que os sentimentos negativos direcionados ao melhor amigo não apresenta diferenças de sexo, pode ser relevante revisar os itens da escala de sentimentos negativos, em especial pelas ressalvas feitas pelos autores no estudo de validação (Souza $\&$ Hutz, 2007a). De outro lado, é possível considerar que os sentimentos negativos percebidos no melhor amigo não sejam a dimensão mais importante para diferenciar homens e mulheres, como alerta Wright (1988). Ademais, está se tratando, no presente estudo e no anterior (Souza \& Hutz, 2007b), da melhor amizade, ou seja, aquela com a qual a pessoa já possui bons níveis de confiança, intimidade e segurança emocional. De todo modo, mais pesquisas sobre a percepção dos sentimentos negativos na amizade de adultos poderão trazer novos direcionamentos.

Na comparação entre estados, apenas os sentimentos negativos associados ao melhor amigo apresentaram diferença significativa. Os participantes mineiros se diferenciaram dos gaúchos nessa avaliação, o que poderia indicar um certo descontentamento com o amigo. No entanto, as avaliações para satisfação com a amizade não diferiram entre estados. $\mathrm{O}$ que pode ocorrer, nesse caso, é uma interferência da duração da amizade. À medida que o relacionamento de amizade se prolonga, os amigos passam a melhor conhecer o outro, passam por uma variedade de experiências, inclusive negativas e com o envolvimento de conflitos entre os dois. As tentativas exitosas de resolução de conflitos fortalecem a amizade e tornam os amigos mais compreensivos e tolerantes, o que pode, inclusive, reduzir a probabilidade futura de envolvimento em novos conflitos. Assim, as amizades gaúchas, que apresentaram duração aproximada de 10 anos, podem estar mais consolidadas e apresentar mais experiência prévia com sentimentos negativos do que as amizades mineiras da amostra, com média de duração de sete anos e com origem predominantemente escolar.
Concordando com a literatura empírica (DeSousa \& Cerqueira-Santos, 2012; Mendelson \& Aboud, 1999; Souza \& Hutz, 2008a), a maioria dos participantes possui uma melhor amizade de mesmo sexo. O vínculo concomitante sugere um apego maior a amizades antigas (escola) em mineiros, o que pode ser explicado pela média de idade inferior à dos participantes gaúchos em quase seis anos, posicionando os primeiros no início da adultez-jovem, e os últimos já dessa etapa se despedindo. Nos estudos com amostras gaúcha e sergipana, a origem da amizade mais citada foi, realmente, colega de escola (DeSousa \& Cerqueira-Santos, 2012; Souza \& Hutz, 2008a).

Foi pequena a diferença entre as amostras gaúcha e mineira quanto à indicação de reciprocidade na amizade (61 e 64\%, respectivamente), e porcentagem semelhante foi encontrada nos trabalhos anteriores $(64 \%$ em Souza \& Hutz, 2008a; 65\% em DeSousa \& Cerqueira-Santos, 2012). Já com relação à maior duração da amizade na amostra do RS, muito provavelmente essa diferença também pode ser explicada em função da diferença etária dos participantes dos dois estados. Os gaúchos, com média de 26 anos de idade, têm condições de envolvimento em uma melhor amizade por mais tempo, no contraste com os mineiros, que com idade ao redor de 20 anos, ainda tendem a indicar uma melhor amizade provavelmente advinda do ensino médio.

Sobre a diferença na quantidade de respostas espontâneas sobre tópicos faltantes sobre a amizade nos questionários, muito provavelmente os adultos-jovens da amostra mineira ainda estão vivenciando ao máximo suas melhores amizades, e a respeito delas ainda têm o que refletir e comentar, portanto listaram mais itens. Rawlins (1992) identifica um ápice desenvolvimental das amizades entre a adolescência e o tempo dedicado ao ensino superior antes dos 30 anos de idade. Sobre as relações indicadas entre amizade, família e romance, trata-se não apenas de uma curiosidade dos participantes, mas também de uma necessidade de mais investimento em pesquisas que estudem conjuntamente a amizade com a família e o romance. Além disso, delineamentos mais sofisticados, que analisem, por exemplo, díades de melhores amigos poderão esclarecer questões de reciprocidade, afinidades e identificação pontuadas pelos respondentes. A inserção da variável personalidade também poderia trazer contribuições nesse sentido, por exemplo, na busca de semelhanças entre melhores amigos.

$\mathrm{Na}$ investigação sobre BES, o primeiro resultado encontrado foi quanto aos gaúchos apresentarem significativamente mais afeto positivo do que os mineiros. Esse resultado poderia estar associado ao índice de desenvolvimento humano municipal disponível até o momento, no qual o RS consta em $9^{\circ}$ lugar (com 0,865$)$ e o MG, em $71^{\circ}$ lugar $(0,839)$ (PNUD, 2013). Isso pode colaborar para a compreensão dos dados. Ademais, para os participantes mineiros o afeto negativo prediz satisfação de vida, enquanto que, para a amostra gaúcha, o afeto positivo e o afeto negativo predizem, juntos, a satisfação de vida. Assim, enquanto para os primeiros a satisfação de vida parece depender da ausência de experiências negativas, para os segundos isso não é suficiente, precisando também de experiências positivas. No entanto, deverão ser desenvolvidos estudos que demonstrem uma tal relação entre 
as aferições subjetiva e objetiva da qualidade de vida de um dado município ou estado brasileiro.

Quanto à questão de gênero no BES, os participantes homens apresentaram significativamente mais afeto negativo e menos satisfação de vida, na comparação com as mulheres. Esse resultado não era esperado, conforme estudos prévios conduzidos no Brasil com as escalas PANAS e de Satisfação de Vida (Giacomoni \& Hutz, 1997). Um novo formato para as escalas PANAS foi desenvolvido por Zanon (2011), que criou sentenças para cada adjetivo da versão original, por exemplo, "Muitas vezes, eu fico nervoso.", no lugar do adjetivo "nervoso". Nesse novo formato, as mulheres apresentaram significativamente mais afetos negativos do que os homens (p. 24), e não houve diferenças para afetos positivos. Assim, o resultado encontrado no presente estudo foi oposto ao de Zanon (2011). Mesmo que se argumente sobre a diferença de formatos das escalas PANAS, há estudos que vêm questionando sua estrutura fatorial. Por exemplo, Gaudreau, Sanchez e Blondin (2006) sugerem uma estrutura com três fatores, no lugar de dois. Portanto, além do alerta que Wright (1988) faz com relação aos estudos sobre amizade, mas que valem para estudos de diferenças gênero em geral, há que se considerar as modificações mais recentes que vem sendo empreendidas nas escalas PANAS (Gaudreau et al., 2006; Zanon, 2011). Portanto, as pesquisas sobre BES e diferenças de sexo precisam de continuidade, aprofundamento e modificações metodológicas com novas amostras brasileiras.

Investigou-se também a relação entre estado civil e BES, com interação significativa entre essas variáveis. Os participantes casados sentem significativamente mais afeto positivo em suas vidas, sem distinção de sexo ou estado. Esse resultado também foi observado por Lansford et al. (2005). A literatura nacional disponível e recente confirma que pessoas casadas tendem a ser mais felizes (Scorsolini-Comin \& Santos, 2010). Todavia não necessariamente se pode, por enquanto, concluir que homens, ao casarem, passam a sentir mais afetos positivos do que negativos. Estudos mais detalhados seriam necessários.

Das relações entre amizade e BES analisadas, percebe-se que a satisfação com a amizade se correlaciona significativamente com satisfação de vida e afetos positivos e negativos, sendo uma relação inversa com este último e positiva com os dois primeiros. Esse resultado se aproxima aos de McDonough e Munz (1994), Rodriguez et al. (2003), Park e Huebner (2005) e Gilman et al. (2008). Esses autores verificaram também a associação positiva e significativa entre bem-estar, felicidade, BES e relações de amizade. Confirma-se, portanto, que a amizade é um dos três relacionamentos mais importantes para a felicidade, como preconizado por Argyle (2001).

A busca por relações através da análise de regressão mostrou que satisfação com a melhor amizade não prediz satisfação de vida, o oposto ao encontrado na literatura empírica revisada, ou seja, de que satisfação com a amizade prediz satisfação de vida. De toda forma, sabe-se que felicidade e BES são dependentes de, principalmente, três diferentes tipos de relacionamentos: família, romance e amizade (Argyle, 2001). Assim, apenas ter bons amigos ou mesmo uma melhor amizade não garante uma felicidade "relacional"; há que se estar contente com as relações com a família e/ou envolvido em um relacionamento romântico que contribua para a felicidade pessoal.

Já se está alcançando, no Brasil, uma quantidade interessante de estudos sobre as relações de amizade, desde a infância até a velhice. Assim, no lugar de sugerir mais estudos sobre amizade no Brasil, há que se buscar investigar novas variáveis relevantes a esse relacionamento, e associá-lo a outros aspectos importantes para a saúde humana, como sua relação com a depressão, a ansiedade, o desempenho no trabalho e, inclusive, seu papel sobre a quantidade e a qualidade de práticas de lazer na vida das pessoas. Outras áreas de conhecimento já vêm investindo no estudo da amizade, como no caso da Administração (Schujmann \& Costa, 2012).

Ainda assim, no que diz respeito à relação entre BES e amizade, há que se investir em novos estudos, mas com o acréscimo da avaliação da qualidade dos relacionamentos românticos e familiares, na busca de um modelo que mapeie, e especifique, as interações possíveis entre os três mais importantes relacionamentos humanos e seu papel na felicidade e BES. Foram essas associações, inclusive, sugeridas pelos participantes do presente estudo, e que, um tanto lentamente, começam a aparecer nos delineamentos de pesquisa em relacionamentos no Brasil.

\section{Referências}

Argyle, M. (2001). The psychology of happiness (2nd ed.). New York: Taylor \& Francis.

Bukowski, W. M., Newcomb, A. F., \& Hartup, W. W. (1996). Friendship and its significance in childhood and adolescence: Introduction and comment. In W. M. Bukowski, A. F. Newcomb, \& W. W. Hartup (Eds.), The company they keep: Friendship in childhood and adolescence (pp. 1-15). Cambridge, UK: University Press.

Carbery, J., \& Buhrmester, D. (1998). Friendship and need fulfillment during three phases of young adulthood. Journal of Social and Personal Relationships, 15(3), 393-409.

Demir, M., Özdemir, M., \& Weitekamp, L. (2007). Looking to happy tomorrows with friends: Best and close relationships as they predict happiness. Journal of Happiness Studies, 8, 243-271.

Demir, M., \& Weitekamp, L. (2007). I am so happy cause today I found my friend: Friendship and personality as predictors of happiness. Journal of Happiness Studies, 8, 181-211.

DeSousa, D. A., \& Cerqueira-Santos, E. (2012). Relacionamentos de amizade íntima entre jovens adultos. Paidéia, 22(53), 325-333.

Diener, E. (1984). Subjective well-being. Psychological Bulletin, 95, 542-575.

Diener, E., Lucas, R., Oishi, S., \& Suh, E. (2002). Looking up and down: Weighting good and bad information in life satisfaction judgments. Personality and Social Psychology Bulletin, 28(4), 437-445.

Diener, E., Emmons, R. A., Larsen, R., \& Griffin, S. (1985). The Satisfaction with Life Scale. Journal of Personality Assessment, 49, 91-95.

Duarte, M., G., \& Souza, L. K. de (2010). O que importa em uma amizade? A percepção de universitários sobre amizades. Interpersona: An International Journal on Personal Relationships, 4(2), 271-290. 
Freire, T., \& Tavares, D. (2011). Influência da autoestima, da regulação emocional e do gênero no bem-estar subjetivo e psicológico de adolescentes. Revista de Psiquiatria Clínica, 38(5), 184-188.

Furman, W., \& Buhrmester, D. (1985). Children's perceptions of the personal relationships in their social networks. Developmental Psychology, 21(6), 1016-1024.

Gaudreau, P., Sanchez, X, \& Blondin, J. (2006). Positive and negative affective states in a performance-related setting: Testing the factorial structure of the PANAS across two samples of French-Canadian participants. European Journal of Psychological Assessment, 22(4), 240-249.

Giacomoni, C. H., \& Hutz, C. S. (1997, Julho). A mensuração do BES: Escala de afeto positivo e negativo e escala de satisfação de vida. In Sociedade Interamericana de Psicologia (Ed.), Anais do XXVI Congresso Interamericano de Psicologia da Sociedade Interamericana de Psicologia, São Paulo (p. 313). São Paulo: Autor.

Giacomoni, C., Hutz, C. S., \& Reppold, C. (2007). BES: Definição e formas de avaliação. In P. Schelini (Ed.), Alguns domínios da avaliação psicológica (pp. 111-124). Campinas: Alínea.

Gilman, R., Huebner, E., Tian, L., Park, N., O’Byrne, J., Schiff, M., Langknecht, H. (2008). Cross-national adolescent multidimensional life satisfaction reports: Analyzes of mean scores and response style differences. Journal of Youth and Adolescence, 37, 142-154.

Kipper, A. (2003). Amizade, trabalho e BES. Dissertação de mestrado não-publicada, Universidade Federal do Rio Grande do Sul, Porto Alegre.

Lansford, J., Antonucci, T., Akiyama, H., \& Takahashi, K. (2005). A quantitative and qualitative approach to social relationships and well-being in the United States and Japan. Journal of Comparative Family Studies, January, 1-22.

Lyubomirsky, S., King, L., \& Diener, E. (2005). The benefits of frequent positive affect: Does happiness lead to success? Psychological Bulletin, 131(6), 803-855.

McDonough, E., \& Munz, D. (1994). General well-being and perceived adult friendship behaviors. Journal of Social Behavior and Personality, 9(4), 743-752.

Mendelson, M. J., \& Aboud, F. E. (1999). Measuring friendship quality in late adolescents and young adults: McGill Friendship Questionnaires (short report). Canadian Journal of Behavioural Science, 31(2), 130-132.

Mendelson, M. J., \& Aboud, F. E. (2003). Measuring friendship quality in late adolescents and young adults: McGill Friendship Questionnaires. Manuscrito não-publicado, McGill University, Montreal, Canada.

Moreira, M. C., \& Sarriera, J. C. (2006). Preditores de saúde e bem-estar psicológico em adolescentes gestantes. Psico-USF, 11(1), 7-15.

Myers, D. (2000). The funds, friends, and faith of happy people. American Psychologist, 55(1), 56-67.

Park, N., \& Huebner, E. (2005). A cross-cultural study of the levels and correlates of life satisfaction among adolescents. Journal of Cross-Cultural Psychology, 36(4), 444-56.

Pavot, W., Diener, E., \& Fujita, F. (1990). Extraversion and happiness. Personality and Individual Differences, 11, 12991306.
Peron, S., Guimarães, L., \& Souza, L. K. de (2010). Amizade na adolescência e a entrada na universidade. Estudos e Pesquisas em Psicologia, 10(3), 664-681.

Programa das Nações Unidas para o Desenvolvimento (2013). Relatórios de desenvolvimento humano. Retrieved from http:// www.pnud.org.br

Rawlins, W. K. (1992). Friendship matters. New York: Aldine de Gruyter.

Rodriguez, N., Mira, C., Myers, H., Morris, J., \& Cardoza, D. (2003). Family or friends: who plays a greater supportive role for Latino college students? Cultural Diversity and Ethnic Minority Psychology, 9(3), 236-250.

Schujmann, A., \& Costa, S. (2012). A influência da promoção nas relações de amizade no ambiente de trabalho. Psico (PUCRS), 43(1), 39-46.

Scorsolini-Comin, F., \& Santos, M. A. dos (2010). Satisfação com a vida e satisfação diádica: Correlações entre construtos de bem-estar. Psico-USF, 15(2), 249-256.

Souza, L. K. de, \& Hutz, C. S. (2007a). A qualidade da amizade: Adaptação e validação dos Questionários McGill. Aletheia, 25, 82-96.

Souza, L. K. de, \& Hutz, C. S. (2007b). Diferenças de gênero na percepção da qualidade da amizade. Psico (PUCRS), 38, $125-132$.

Souza, L. K. de, \& Hutz, C. S. (2008a). Amizade na adultez: Fatores individuais, ambientais, situacionais e diádicos. Interação em Psicologia, 12(1), 77-85.

Souza, L. K. de, \& Hutz, C. S. (2008b). Relacionamentos pessoais e sociais: Amizade em adultos. Psicologia em Estudo, 13(2), 257-265.

Watson, D., Clark, L. A., \& Tellegen, A. (1988). Development and validation of brief measures of positive and negative affect: The PANAS scales. Journal of Personality and Social Psychology, 54, 1063-1070.

Woyciekoski, C., Stenert, F., \& Hutz, C. S. (2012). Determinantes do bem-estar subjetivo. Psico (PUCRS), 43(3), 280-288.

Wright, P. (1988). Interpreting research on gender differences in friendship: A case for moderation and a plea for caution. Journal of Social and Personal Relationships, 5(3), 367-373.

Zanon, C. (2011). Construção de uma escala de afetos e suas relações com os cinco grandes fatores da personalidade (Big Five). Tese de doutorado não-publicada. Universidade Federal do Rio Grande do Sul, Porto Alegre. 